\title{
A FOURIER APPROACH FOR THE LEVEL CROSSINGS OF SHOT NOISE PROCESSES WITH JUMPS
}

\author{
HERMINE BIERMÉ* AND \\ AGNÈS DESOLNEUX, ${ }^{* *}$ Université Paris Descartes
}

\begin{abstract}
We use a change-of-variable formula in the framework of functions of bounded variation to derive an explicit formula for the Fourier transform of the level crossing function of shot noise processes with jumps. We illustrate the result in some examples and give some applications. In particular, it allows us to study the asymptotic behavior of the mean number of level crossings as the intensity of the Poisson point process of the shot noise process goes to infinity.
\end{abstract}

Keywords: Shot noise process; level crossing; stationary process; Poisson point process; characteristic function; functions of bounded variation; change-of-variable formula

2010 Mathematics Subject Classification: Primary 60G17

Secondary 60E10; 26A45; 60G10; 60F05

\section{Introduction}

In this paper we consider a shot noise process which is a real-valued random process given by

$$
X(t)=\sum_{i \in \mathbb{Z}} \beta_{i} g\left(t-\tau_{i}\right), \quad t \in \mathbb{R},
$$

where $g$ is a given (deterministic) measurable function (it will be called the kernel function of the shot noise), the $\left\{\tau_{i}\right\}_{i \in \mathbb{Z}}$ are the points of a homogeneous Poisson point process on the line of intensity $\lambda>0$, and the $\left\{\beta_{i}\right\}_{i \in \mathbb{Z}}$ are independent copies of a random variable $\beta$ (called the impulse), independent of $\left\{\tau_{i}\right\}_{i \in \mathbb{Z} \text {. }}$

Such a process has many applications (see [16] and the references therein for instance), and it is a well-known and well-studied mathematical model (see [5], [10], and [15] for some of its properties).

We will be interested here in the level crossings of such a process. Usually, the mean number of level crossings of a stochastic process is computed thanks to Rice's formula (see [1] or [12]), which requires some regularity conditions on the joint probability density of $X$ and of its derivative. This joint probability density is generally not easy to obtain or to study. Its existence is also sometimes in question. This is why, instead of working directly with the mean number of level crossings, we will work with the Fourier transform of the function that maps each level $\alpha$ to the mean number of crossings of the level $\alpha$ per unit length. Thanks to a change-of-variable formula, we relate this Fourier transform to the characteristic function of the shot noise process (which, unlike the probability density, always exists and is explicit).

Received 25 November 2010; revision received 22 August 2011.

* Postal address: MAP5 (UMR CNRS 8145), Université Paris Descartes, 45 rue des Saints-Pères, 75006 Paris, France.

** Email address: agnes.desolneux@mi.parisdescartes.fr 


\section{General result}

In [3] we studied the level crossings of the shot noise process $X$ when the kernel function $g$ is smooth on $\mathbb{R}$. In this paper we will consider the case where $g$ is a piecewise smooth function, which is not necessarily continuous. We first introduce some definitions and notation.

Let $I$ be an open interval of $\mathbb{R}$, and let $k \geq 0$ be an integer. A function $f: I \rightarrow \mathbb{R}$ is said to be piecewise $C^{k}$ on $I$ if there exists a finite set of points of discontinuity of $f$ on $I$, denoted by

$$
S_{f}=\left\{s_{1}, s_{2}, \ldots, s_{m}\right\} \quad \text { with } m \geq 1 \text { and } s_{1}<\cdots<s_{m},
$$

called the jump set of $f$ on $I$, so that $f$ is of class $C^{k}$ at any point $s$ of $I$ such that $s \notin S_{f}$. We moreover assume that $f$ admits finite left and right limits at each point of $S_{f}$. For a point $s \in I$, we denote by

$$
f(s+)=\lim _{t \rightarrow s, t>s} f(t) \quad \text { and } \quad f(s-)=\lim _{t \rightarrow s, t<s} f(t)
$$

the respective right and left limits of $f$ at $s$. Note that when $s \notin S_{f}$, we simply have $f(s+)=$ $f(s-)=f(s)$. We will also use the following notation: for all $s \in I$,

$$
f^{*}(s):=\max \{f(s+), f(s-)\}, \quad f_{*}(s):=\min \{f(s+), f(s-)\},
$$

and

$$
\Delta f(s):=f(s+)-f(s-) .
$$

Finally, when $k \geq 1$ or $k \geq 2$, we will respectively denote by $f^{\prime}$ and $f^{\prime \prime}$ the functions that are defined at all points $s \notin S_{f}$ by the usual derivatives $f^{\prime}(s)$ and $f^{\prime \prime}(s)$.

In the sequel we will need assumptions on the kernel function $g$ and on the impulse $\beta$ of the shot noise process $X$ defined by (1). These assumptions are grouped together into the following condition:

(C) $\mathrm{E}(|\beta|)<\infty, g$ is piecewise $C^{2}$ on $\mathbb{R}$, and $g, g^{\prime}, g^{\prime \prime} \in L^{1}(\mathbb{R})$.

We will also denote the jump set of $g$ on $\mathbb{R}$ by

$$
S_{g}=\left\{t_{1}, t_{2}, \ldots, t_{n}\right\} \quad \text { with } t_{1}<\cdots<t_{n} .
$$

As a consequence of $(\mathrm{C}), g$ has finite total variation on $\mathbb{R}$, which means that

$$
\mathrm{TV}(g, \mathbb{R})=\sup _{P} \sum_{k=1}^{n_{P}}\left|g\left(a_{k}\right)-g\left(a_{k-1}\right)\right|=\int_{\mathbb{R}}\left|g^{\prime}(s)\right| \mathrm{d} s+\sum_{j=1}^{n}\left|\Delta g\left(t_{j}\right)\right|<\infty,
$$

where the supremum is taken over all partitions $P=\left\{a_{0}, \ldots, a_{n_{P}}\right\}$ of $\mathbb{R}$ with $n_{P} \geq 1$ and $a_{0}<\cdots<a_{n_{P}}$.

Finally, we assume that the points $\left\{\tau_{i}\right\}$ of the Poisson point process are indexed by points from $\mathbb{Z}$ in such a way that, for any $k \in \mathbb{N}$, we have $0<\tau_{0}<\tau_{1}<\tau_{2}<\cdots<\tau_{k}<\cdots$ and $0>\tau_{-1}>\tau_{-2}>\cdots>\tau_{-k}>\cdots$.

\subsection{Piecewise regularity of the shot noise process}

The shot noise process 'inherits' the regularity of the kernel function $g$. More precisely, we have the following result. 
Theorem 1. Assume that condition $(C)$ holds. Then the shot noise process $X$ defined by (1) is a stationary process which is almost surely piecewise $C^{1}$ on any interval $(a, b)$ of $\mathbb{R}$. The jump set of $X$ on $(a, b)$ is

$$
S_{X} \cap(a, b), \quad \text { where } S_{X}=\bigcup_{i \in \mathbb{Z}}\left(\tau_{i}+S_{g}\right),
$$

and, for all $t \notin S_{X}$,

$$
X^{\prime}(t)=\sum_{i \in \mathbb{Z}} \beta_{i} g^{\prime}\left(t-\tau_{i}\right)
$$

Proof. Note that, since $\mathrm{E}(|\beta|)<\infty$ and $g \in L^{1}(\mathbb{R})$, for any $t \in \mathbb{R}$, the random variable $X(t)$ is well defined and $\mathrm{E}(X(t))=\lambda \mathrm{E}(\beta) \int_{\mathbb{R}} g(s) \mathrm{d} s$, according to [16]. Moreover, since the Poisson point process is homogeneous, $X$ is a stationary process.

Let us first remark that since the jump set $S_{g}$ of $g$ contains exactly $n$ points, we can write $g$ as the sum of $2 n$ piecewise $C^{2}$ functions on $\mathbb{R}$, each of them having only one discontinuity point and having the same regularity properties as $g$. Therefore, we may and will assume that $g$ has only one discontinuity point and we write $S_{g}=\left\{t_{1}\right\}$. Let $i_{0} \in \mathbb{Z}$. We set $I_{i_{0}}:=\left[t_{1}+\tau_{i_{0}}, t_{1}+\tau_{i_{0}+1}\right]$. Then, for any $t \in \mathbb{R}$,

$$
X(t)=\sum_{i>i_{0}+1} \beta_{i} g\left(t-\tau_{i}\right)+\sum_{i<i_{0}} \beta_{i} g\left(t-\tau_{i}\right)+\beta_{i_{0}} g\left(t-\tau_{i_{0}}\right)+\beta_{i_{0}+1} g\left(t-\tau_{i_{0}+1}\right) .
$$

The function $t \mapsto g(t-s)$ is $C^{2}$ on $I_{i_{0}}$ for any $s<\tau_{i_{0}}$ so that, almost surely (a.s.), for any $i<i_{0}$, the function $t \mapsto g\left(t-\tau_{i}\right)$ is $C^{2}$ on $I_{i_{0}}$ with $g\left(\left(t_{1}+\tau_{i_{0}}-\tau_{i}\right)+\right)=g\left(\left(t_{1}+\tau_{i_{0}}-\tau_{i}\right)-\right)$. Moreover,

$$
\mathrm{E}\left(\sum_{i<i_{0}}\left|\beta_{i}\right| \sup _{t \in I_{i_{0}}}\left|g^{\prime}\left(t-\tau_{i}\right)\right| \mid \tau_{i_{0}}, \tau_{i_{0}+1}\right)=\lambda \mathrm{E}(|\beta|) \int_{-\infty}^{0} \sup _{t \in I_{i_{0}}}\left|g^{\prime}\left(t-s-\tau_{i_{0}}\right)\right| \mathrm{d} s,
$$

using the fact that $\left\{\tau_{i}-\tau_{i_{0}} ; i<i_{0}\right\}$ is a homogeneous Poisson point process with intensity $\lambda$ on $(-\infty, 0)$, and independent of $\tau_{i_{0}}$ and $\tau_{i_{0}+1}$. Now, for any $t \in I_{i_{0}}$ and $s<0$,

$$
g^{\prime}\left(t-s-\tau_{i_{0}}\right)=\int_{t_{1}+\tau_{i_{0}}}^{t} g^{\prime \prime}\left(u-s-\tau_{i_{0}}\right) \mathrm{d} u+g^{\prime}\left(t_{1}-s\right),
$$

so that, by the Fubini-Tonelli theorem,

$$
\int_{-\infty}^{0} \sup _{t \in I_{i_{0}}}\left|g^{\prime}\left(t-s-\tau_{i_{0}}\right)\right| \mathrm{d} s \leq\left(\tau_{i_{0}+1}-\tau_{i_{0}}\right) \int_{\mathbb{R}}\left|g^{\prime \prime}(s)\right| \mathrm{d} s+\int_{\mathbb{R}}\left|g^{\prime}(s)\right| \mathrm{d} s .
$$

Then

$$
\mathrm{E}\left(\sum_{i<i_{0}}\left|\beta_{i}\right| \sup _{t \in I_{i_{0}}}\left|g^{\prime}\left(t-\tau_{i}\right)\right|\right) \leq \lambda \mathrm{E}(|\beta|)\left(\frac{1}{\lambda} \int_{\mathbb{R}}\left|g^{\prime \prime}(s)\right| \mathrm{d} s+\int_{\mathbb{R}}\left|g^{\prime}(s)\right| \mathrm{d} s\right)<\infty,
$$

so that, a.s., the series $t \mapsto \sum_{i<i_{0}} \beta_{i} g^{\prime}\left(t-\tau_{i}\right)$ is uniformly convergent on $I_{i_{0}}$. Therefore, a.s., the series $t \mapsto \sum_{i<i_{0}} \beta_{i} g\left(t-\tau_{i}\right)$ is continuously differentiable on $I_{i_{0}}$ with

$$
\left(\sum_{i<i_{0}} \beta_{i} g\left(t-\tau_{i}\right)\right)^{\prime}=\sum_{i<i_{0}} \beta_{i} g^{\prime}\left(t-\tau_{i}\right)
$$

and $\sum_{i<i_{0}} \beta_{i} g\left(\left(t_{1}+\tau_{i_{0}}-\tau_{i}\right)+\right)=\sum_{i<i_{0}} \beta_{i} g\left(\left(t_{1}+\tau_{i_{0}}-\tau_{i}\right)-\right)$. 
The same proof applies for $\sum_{i>i_{0}+1} \beta_{i} g\left(t-\tau_{i}\right)$. To conclude, it is sufficient to remark that, for $i \in\left\{i_{0}, i_{0}+1\right\}$, the function $t \mapsto g\left(t-\tau_{i}\right)$ is continuously differentiable in the interior of $I_{i_{0}}$. Moreover, $g\left(\left(t_{1}+\tau_{i_{0}}-\tau_{i_{0}+1}\right)+\right)=g\left(\left(t_{1}+\tau_{i_{0}}-\tau_{i_{0}+1}\right)-\right), g\left(\left(t_{1}+\tau_{i_{0}}-\tau_{i_{0}}\right)+\right)=g\left(t_{1}+\right)$, and $g\left(\left(t_{1}+\tau_{i_{0}}-\tau_{i_{0}}\right)-\right)=g\left(t_{1}-\right)$. Finally, a.s., $X$ is continuously differentiable in the interior of $I_{i_{0}}$ with

$$
X^{\prime}(t)=\sum_{i} \beta_{i} g^{\prime}\left(t-\tau_{i}\right)
$$

and $X\left(\left(t_{1}+\tau_{i_{0}}\right)+\right)-X\left(\left(t_{1}+\tau_{i_{0}}\right)-\right)=\beta_{i_{0}}\left(g\left(t_{1}+\right)-g\left(t_{1}-\right)\right)$. This completes the proof of the theorem since $\mathbb{R}=\bigcup_{i_{0} \in \mathbb{Z}} I_{i_{0}}$.

Remark 1. Theorem 1 implies that, under condition (C), the shot noise process $X$ a.s. has a finite total variation on any interval $(a, b)$ of $\mathbb{R}$. By stationarity we can focus on what happens on the interval $(0,1)$. Then $X$ a.s. has a finite number of points of discontinuity in $(0,1)$ and its total variation on $(0,1)$ is given by

$$
\operatorname{TV}(X,(0,1))=\int_{0}^{1}\left|X^{\prime}(t)\right| \mathrm{d} t+\sum_{j=1}^{n} \sum_{\tau_{i} \in\left(-t_{j}, 1-t_{j}\right)}\left|\beta_{i}\right|\left|\Delta g\left(t_{j}\right)\right|
$$

\subsection{Level crossings}

We start this section with a general definition and a result on the level crossings of a piecewise smooth function.

Let $f$ be a piecewise $C^{1}$ function on an interval $(a, b)$ of $\mathbb{R}$. We can define its level crossings on $(a, b)$ by considering, for any level $\alpha \in \mathbb{R}$,

$$
N_{f}(\alpha,(a, b))=\#\left\{s \in(a, b) ; f_{*}(s) \leq \alpha \leq f^{*}(s)\right\} \in \mathbb{N} \cup\{\infty\},
$$

where we have used the notation given in (2) and \#\{.\} to denote the number of elements of the set $\{\cdot\}$. The number $N_{f}(\alpha,(a, b))$ may be infinite. This is, for instance, what happens if there exists a subinterval of $(a, b)$ on which $f$ is constant and equal to $\alpha$ (the value $\alpha$ is then called a critical value of $f$ ). But, according to the Morse-Sard theorem (see [13, p. 10]), the set of these critical values has Lebesgue measure 0 . Let us also mention that a weak variant of the Morse-Sard theorem for Lipschitz functions can be found in [8, p. 112].

Then, a change-of-variable formula for piecewise $C^{1}$ functions is obtained in the following proposition.

Proposition 1. Let $a, b \in \mathbb{R}$ with $a<b$ and $f$ be a piecewise $C^{1}$ function on $(a, b)$. Then, for any bounded continuous function $h$ defined on $\mathbb{R}$,

$$
\int_{\mathbb{R}} h(\alpha) N_{f}(\alpha,(a, b)) \mathrm{d} \alpha=\int_{a}^{b} h(f(s))\left|f^{\prime}(s)\right| \mathrm{d} s+\sum_{s \in S_{f} \cap(a, b)} \int_{f_{*}(s)}^{f^{*}(s)} h(\alpha) \mathrm{d} \alpha .
$$

Proof. Let us assume that $S_{f} \cap(a, b)=\left\{s_{j} ; 1 \leq j \leq m\right\}$ with $m \geq 1$ and $s_{0}:=a<s_{1}<$ $\cdots<s_{m}<s_{m+1}:=b$. Then

$$
N_{f}(\alpha,(a, b))=\sum_{j=0}^{m} \#\left\{s \in\left(s_{j}, s_{j+1}\right) ; f(s)=\alpha\right\}+\sum_{j=1}^{m} \mathbf{1}_{\left[f_{*}\left(s_{j}\right), f^{*}\left(s_{j}\right)\right]}(\alpha) .
$$


Let $h$ be a bounded continuous function on $\mathbb{R}$. According to the change-of-variable formula for Lipschitz functions (see [8, p. 99]), for any $j=0, \ldots, m$, we have

$$
\int_{\mathbb{R}} h(\alpha) \#\left\{s \in\left(s_{j}, s_{j+1}\right) ; f(s)=\alpha\right\} \mathrm{d} \alpha=\int_{s_{j}}^{s_{j+1}} h(f(s))\left|f^{\prime}(s)\right| \mathrm{d} s .
$$

Summing these equalities over $j=0, \ldots, m$, and using (4), completes the proof.

Note that a change-of-variable formula for functions of bounded variation was also obtained by Choski and Fonseca [6]. However, their formula is valid away from the jump set of $f$, whereas (3) explicitly takes into account the contribution of the jump set. Let us also mention the fact that (3) is a kind of extension of Banach's theorem used by Rychlik [17, p. 335]. The main difference between our approach and that of Rychlik is that (3) involves a function $h$ that is not necessarily constant; however, we restrict ourselves to a piecewise $C^{1}$ function $f$, whereas Banach's theorem is valid for functions of bounded variation.

Now we consider the shot noise process $X$. For $\alpha \in \mathbb{R}$, let $N_{X}(\alpha)$ be the random variable that counts the number of crossings of the level $\alpha$ by the process $X$ in the interval $(0,1)$. Using (2), it is defined by

$$
N_{X}(\alpha)=\#\left\{t \in(0,1) ; X_{*}(t) \leq \alpha \leq X^{*}(t)\right\} .
$$

We will be mainly interested in its expectation, namely in

$$
C_{X}(\alpha)=\mathrm{E}\left(N_{X}(\alpha)\right) .
$$

The function $\alpha \mapsto C_{X}(\alpha)$ is called the mean level crossing function and we compute its Fourier transform in the following theorem. This result has to be related to the heuristic approach of Bar-David and Nemirovsky [2] (in particular their Equation (13), in which the joint density of $X(t)$ and $X^{\prime}(t)$ is given by Rice's formula, whose validity was not checked by Bar-David and Nemirovsky). Our theorem will involve the characteristic function of the shot noise process, which is easily computable. Actually, if we define, for all $u, v \in \mathbb{R}$,

$$
\psi(u, v)=\mathrm{E}\left(\mathrm{e}^{\mathrm{i} u X(0)+\mathrm{i} v X^{\prime}(0)}\right) \quad \text { and } \quad \hat{F}(u)=\mathrm{E}\left(\mathrm{e}^{\mathrm{i} u \beta}\right),
$$

then it is well known (see [16] for instance) that

$$
\psi(u, v)=\exp \left(\lambda \int_{\mathbb{R}}\left(\hat{F}\left(u g(s)+v g^{\prime}(s)\right)-1\right) \mathrm{d} s\right) .
$$

Theorem 2. Assume that condition $(C)$ is satisfied. Then the mean level crossing function $C_{X}$ belongs to $L^{1}(\mathbb{R})$ and

$$
\int_{\mathbb{R}} C_{X}(\alpha) \mathrm{d} \alpha=\mathrm{E}(\operatorname{TV}(X,(0,1))) \leq \lambda \mathrm{E}(|\beta|) \operatorname{TV}(g, \mathbb{R}) .
$$

Moreover, its Fourier transform, denoted by $u \mapsto \widehat{C_{X}}(u)$, is given by

$$
\widehat{C_{X}}(u)=\mathrm{E}\left(\mathrm{e}^{\mathrm{i} u X(0)}\left|X^{\prime}(0)\right|\right)+\lambda \mathrm{E}\left(\mathrm{e}^{\mathrm{i} u X(0)}\right) \frac{1}{\mathrm{i} u} \sum_{j=1}^{n}\left(\mathrm{E}\left(\mathrm{e}^{\mathrm{i} u(\beta g)^{*}\left(t_{j}\right)}\right)-\mathrm{E}\left(\mathrm{e}^{\mathrm{i} u(\beta g)_{*}\left(t_{j}\right)}\right)\right)
$$

for $u \neq 0$ and by

$$
\widehat{C_{X}}(0)=\mathrm{E}(\operatorname{TV}(X,(0,1)))=\mathrm{E}\left(\left|X^{\prime}(0)\right|\right)+\lambda \mathrm{E}(|\beta|) \sum_{j=1}^{n}\left|\Delta g\left(t_{j}\right)\right|
$$

for $u=0$. 
Proof. According to Theorem 1, we can apply Proposition 1 so that, a.s., for any bounded continuous function $h$ defined on $\mathbb{R}$,

$$
\int_{\mathbb{R}} h(\alpha) N_{X}(\alpha) \mathrm{d} \alpha=\int_{0}^{1} h(X(t))\left|X^{\prime}(t)\right| \mathrm{d} t+\sum_{t \in S_{X} \cap(0,1)} \int_{X_{*}(t)}^{X^{*}(t)} h(\alpha) \mathrm{d} \alpha .
$$

Taking $h=1$, we obtain

$$
\int_{\mathbb{R}} N_{X}(\alpha) \mathrm{d} \alpha=\int_{0}^{1}\left|X^{\prime}(t)\right| \mathrm{d} t+\sum_{t \in S_{X} \cap(0,1)}|\Delta X(t)|=\operatorname{TV}(X,(0,1)) .
$$

Using the stationarity of $X^{\prime}$, we have $\mathrm{E}\left(\int_{0}^{1}\left|X^{\prime}(t)\right| \mathrm{d} t\right)=\mathrm{E}\left(\left|X^{\prime}(0)\right|\right) \leq \lambda \mathrm{E}(|\beta|) \int_{\mathbb{R}}\left|g^{\prime}(s)\right| \mathrm{d} s$ and

$$
\mathrm{E}\left(\sum_{t \in S_{X} \cap(0,1)}|\Delta X(t)|\right)=\lambda \mathrm{E}(|\beta|) \sum_{j=1}^{n}\left|\Delta g\left(t_{j}\right)\right| .
$$

Therefore,

$$
\int_{\mathbb{R}} C_{X}(\alpha) \mathrm{d} \alpha \leq \lambda \mathrm{E}(|\beta|)\left(\int_{\mathbb{R}}\left|g^{\prime}(s)\right| \mathrm{d} s+\sum_{j=1}^{n}\left|\Delta g\left(t_{j}\right)\right|\right) \leq \lambda \mathrm{E}(|\beta|) \mathrm{TV}(g, \mathbb{R}) .
$$

Now, taking $h(\alpha)=\mathrm{e}^{\mathrm{i} u \alpha}$ for some $u \in \mathbb{R}$ with $u \neq 0$ in (7), we obtain

$$
\begin{aligned}
\int_{\mathbb{R}} \mathrm{e}^{\mathrm{i} u \alpha} N_{X}(\alpha) \mathrm{d} \alpha & =\int_{0}^{1} \mathrm{e}^{\mathrm{i} u X(t)}\left|X^{\prime}(t)\right| \mathrm{d} t+\sum_{t \in S_{X} \cap(0,1)} \int_{X_{*}(t)}^{X^{*}(t)} \mathrm{e}^{\mathrm{i} u \alpha} \mathrm{d} \alpha \\
& =\int_{0}^{1} \mathrm{e}^{\mathrm{i} u X(t)}\left|X^{\prime}(t)\right| \mathrm{d} t+\sum_{j=1}^{n} A(j),
\end{aligned}
$$

where

$$
A(j):=\frac{1}{\mathrm{i} u} \sum_{\tau_{i} \in\left(-t_{j}, 1-t_{j}\right)} \mathrm{e}^{\mathrm{i} u X\left(\left(t_{j}+\tau_{i}\right)+\right)}\left(\mathrm{e}^{\mathrm{i} u \max \left(\beta_{i} \Delta g\left(t_{j}\right), 0\right)}-\mathrm{e}^{\mathrm{i} u \min \left(\beta_{i} \Delta g\left(t_{j}\right), 0\right)}\right) .
$$

Now, let us write $X\left(\left(t_{j}+\tau_{i}\right)+\right)=\sum_{\tau_{k} \neq \tau_{i}} \beta_{k} g\left(t_{j}+\tau_{i}-\tau_{k}\right)+\beta_{i} g\left(t_{j}+\right)$ and

$$
B\left(u, t_{j}, \beta_{i}\right):=\frac{1}{\mathrm{i} u}\left(\mathrm{e}^{\mathrm{i} u\left(\beta_{i} g\right)^{*}\left(t_{j}\right)}-\mathrm{e}^{\mathrm{i} u\left(\beta_{i} g\right)_{*}\left(t_{j}\right)}\right),
$$

such that

$$
A(j)=\sum_{\tau_{i} \in\left(-t_{j}, 1-t_{j}\right)} \exp \left(\mathrm{i} u \sum_{k \neq i} \beta_{k} g\left(t_{j}+\tau_{i}-\tau_{k}\right)\right) B\left(u, t_{j}, \beta_{i}\right) .
$$

Then, for $M>\max _{1 \leq j \leq n}\left|t_{j}\right|+1$, we consider

$$
A_{M}(j)=\sum_{\tau_{i} \in\left(-t_{j}, 1-t_{j}\right)} \exp \left(\mathrm{i} u \sum_{k \neq i,\left|\tau_{k}\right| \leq M} \beta_{k} g\left(t_{j}+\tau_{i}-\tau_{k}\right)\right) B\left(u, t_{j}, \beta_{i}\right)
$$


such that $A_{M}(j) \rightarrow A(j)$ a.s. as $M \rightarrow+\infty$. We have

$$
A_{M}(j) \stackrel{\mathrm{D}}{=} \sum_{i=1}^{N_{M}} \mathbf{1}_{\left(-t_{j}, 1-t_{j}\right)}\left(U_{i}\right) \exp \left(\mathrm{i} u \sum_{k=1, k \neq i}^{N_{M}} \beta_{k} g\left(t_{j}+U_{i}-U_{k}\right)\right) B\left(u, t_{j}, \beta_{i}\right),
$$

where $\left(U_{k}\right)_{k \in \mathbb{N}}$ is an independent and identically distributed sequence of random variables of uniform law on $[-M, M]$ independent of $\left(\beta_{k}\right)_{k \in \mathbb{N}}$, and $N_{M}$ is a Poisson random variable of parameter $2 \lambda M$, independent of $\left(U_{k}\right)_{k \in \mathbb{N}}$ and $\left(\beta_{k}\right)_{k \in \mathbb{N}}$. We adopt the convention that $\sum_{i=1}^{0}=0$. Now, by conditioning we obtain

$$
\mathrm{E}\left(A_{M}(j)\right)=\sum_{m=0}^{+\infty} \mathrm{E}\left(A_{M}(j) \mid N_{M}=m\right) \mathrm{P}\left[N_{M}=m\right],
$$

and then computing $\mathrm{E}\left(A_{M}(j) \mid N_{M}=m\right)$ for all $m \geq 0$, using the independence of $\left(U_{k}\right)_{k \in \mathbb{N}}$ and $\left(\beta_{k}\right)_{k \in \mathbb{N}}$, finally leads to

$$
\mathrm{E}\left(A_{M}(j)\right)=\lambda \mathrm{E}\left(B\left(u, t_{j}, \beta\right)\right) \int_{-t_{j}}^{1-t_{j}} \exp \left(\lambda \int_{-M+x+t_{j}}^{M+x+t_{j}}(\hat{F}(u g(s))-1) \mathrm{d} s\right) \mathrm{d} x,
$$

where $\hat{F}$ is given by (5). As a consequence, using Lebesgue's theorem and (6) for $\mathrm{E}\left(\mathrm{e}^{\mathrm{i} u X(0)}\right)$, we obtain

$$
\mathrm{E}(A(j))=\lambda \mathrm{E}\left(\mathrm{e}^{\mathrm{i} u X(0)}\right) \mathrm{E}\left(B\left(u, t_{j}, \beta\right)\right)
$$

Finally, taking the expectation of both sides of (8) and using the stationarity of $X$ leads to the announced result for $\widehat{C_{X}}(u)$.

Proposition 2. Under condition $(C)$, if we assume moreover that $\mathrm{E}\left(\beta^{2}\right)<\infty$ and that $g^{\prime} \in$ $L^{2}(\mathbb{R})$, then, for $u \neq 0$,

$$
\begin{aligned}
\widehat{C_{X}}(u)= & \frac{-1}{\pi} \int_{0}^{+\infty} \frac{1}{v}\left(\frac{\partial \psi}{\partial v}(u, v)-\frac{\partial \psi}{\partial v}(u,-v)\right) \mathrm{d} v \\
& +\lambda \psi(u, 0) \frac{1}{\mathrm{i} u} \sum_{j=1}^{n}\left(\mathrm{E}\left(\mathrm{e}^{\mathrm{i} u(\beta g)^{*}\left(t_{j}\right)}\right)-\mathrm{E}\left(\mathrm{e}^{\mathrm{i} u(\beta g)_{*}\left(t_{j}\right)}\right)\right),
\end{aligned}
$$

where $\psi$ is given by (5).

Proof. Since $g^{\prime} \in L^{1}(\mathbb{R})$ and since $g$ has a finite number of discontinuity points with finite left and right limits, it follows that $g \in L^{\infty}(\mathbb{R})$. Consequently, $g \in L^{\infty}(\mathbb{R}) \cap L^{1}(\mathbb{R}) \subset L^{2}(\mathbb{R})$. Therefore, when $\mathrm{E}\left(\beta^{2}\right)<\infty$ and $g^{\prime} \in L^{2}(\mathbb{R})$, the characteristic function $\psi$ of $\left(X(0), X^{\prime}(0)\right)$ is $C^{2}$ on $\mathbb{R}^{2}$. Then, $\int_{0}^{+\infty}(1 / v)(\partial \psi(u, v) / \partial v-\partial \psi(u,-v) / \partial v) \mathrm{d} v$ is well defined and is the Hilbert transform of the function $v \mapsto \partial \psi(u, v) / \partial v$. Moreover, the computations of Theorem 1 in our previous paper [3] yield

$$
\mathrm{E}\left(\mathrm{e}^{\mathrm{i} u X(0)}\left|X^{\prime}(0)\right|\right)=\frac{-1}{\pi} \int_{0}^{+\infty} \frac{1}{v}\left(\frac{\partial \psi}{\partial v}(u, v)-\frac{\partial \psi}{\partial v}(u,-v)\right) \mathrm{d} v .
$$

This completes the proof. 


\section{A particular case}

The formula for $\widehat{C_{X}}(u)$ can become simpler in some cases. The first particular case is when the kernel $g$ is piecewise constant, since then $X^{\prime}(0)=0$ a.s. and, thus, the term $\mathrm{E}\left(\mathrm{e}^{\mathrm{i} u X(0)}\left|X^{\prime}(0)\right|\right)$ vanishes. Let us also make the following remark.

Remark 2. When $\beta \geq 0$ a.s., then, for any $h_{1}<h_{2} \in \mathbb{R}$, the function

$$
u \mapsto \frac{1}{\mathrm{i} u} \psi(u, 0)\left(\hat{F}\left(u h_{2}\right)-\hat{F}\left(u h_{1}\right)\right)
$$

is the Fourier transform of the function $\alpha \mapsto \mathrm{P}\left[\alpha-\beta h_{2} \leq X(0) \leq \alpha-\beta h_{1}\right]$, where $\beta$ and $X(0)$ are taken to be independent.

We now give a simpler formula for $\widehat{C_{X}}(u)$ in the case where $g$ is piecewise nonincreasing (meaning that $g^{\prime} \leq 0$ and, thus, $g$ is nonincreasing on each of the intervals on which it is continuous, but it can have jumps $t_{j}$ such that $\left.\Delta g\left(t_{j}\right)=g\left(t_{j}+\right)-g\left(t_{j}-\right)>0\right)$. In that case, we have the following proposition.

Proposition 3. Assume that condition (C) holds. Assume moreover that $\beta \geq 0$ a.s. and that $g^{\prime} \leq 0$. Then, for all $u \in \mathbb{R}$,

$$
\widehat{C_{X}}(u)=2 \lambda \psi(u, 0) \frac{1}{\mathrm{i} u} \sum_{\left\{t_{j}: \Delta g\left(t_{j}\right)>0\right\}}\left(\hat{F}\left(u g\left(t_{j}+\right)\right)-\hat{F}\left(u g\left(t_{j}-\right)\right)\right),
$$

where $\psi$ and $\hat{F}$ are given by (5). As a consequence, for almost every $\alpha \in \mathbb{R}$,

$$
C_{X}(\alpha)=2 \lambda \sum_{\left\{t_{j}: \Delta g\left(t_{j}\right)>0\right\}} \mathrm{P}\left[\alpha-\beta g\left(t_{j}+\right) \leq X(0) \leq \alpha-\beta g\left(t_{j}-\right)\right],
$$

where $\beta$ and $X(0)$ are taken to be independent.

Proof. Since $g^{\prime} \leq 0$, we have $X^{\prime}(0) \leq 0$ a.s. and, consequently,

$$
\mathrm{E}\left(\mathrm{e}^{\mathrm{i} u X(0)}\left|X^{\prime}(0)\right|\right)=-\mathrm{E}\left(\mathrm{e}^{\mathrm{i} u X(0)} X^{\prime}(0)\right)=\mathrm{i} \frac{\partial \psi}{\partial v}(u, 0)
$$

Now, since $g$ is piecewise nonincreasing and belongs to $L^{1}(\mathbb{R})$, we have $\lim _{|s| \rightarrow+\infty} g(s)=0$ and, thus, $\lim _{|s| \rightarrow+\infty} \hat{F}(u g(s))=1$. Then, using $(6)$ for $\psi(u, v)$, we obtain

$$
\begin{aligned}
& \frac{\partial \psi}{\partial v}(u, 0)=\lambda \psi(u, 0) \int_{\mathbb{R}} g^{\prime}(s) \hat{F}^{\prime}(u g(s)) \mathrm{d} s \\
& =\lambda \psi(u, 0)\left(\int_{-\infty}^{t_{1}} g^{\prime}(s) \hat{F}^{\prime}(u g(s)) \mathrm{d} s\right. \\
& \left.+\sum_{j=1}^{n-1} \int_{t_{j}}^{t_{j+1}} g^{\prime}(s) \hat{F}^{\prime}(u g(s)) \mathrm{d} s+\int_{t_{n}}^{+\infty} g^{\prime}(s) \hat{F}^{\prime}(u g(s)) \mathrm{d} s\right)
\end{aligned}
$$




$$
\begin{aligned}
& =\lambda \psi(u, 0) \frac{1}{u}\left(\hat{F}\left(u g\left(t_{1}-\right)\right)+\sum_{j=1}^{n-1}\left(\hat{F}\left(u g\left(t_{j+1}-\right)\right)-\hat{F}\left(u g\left(t_{j}+\right)\right)\right)\right. \\
& \left.-\hat{F}\left(u g\left(t_{n}+\right)\right)\right) \\
& =\lambda \psi(u, 0) \frac{1}{u} \sum_{j=1}^{n}\left(\hat{F}\left(u g\left(t_{j}-\right)\right)-\hat{F}\left(u g\left(t_{j}+\right)\right)\right) .
\end{aligned}
$$

Consequently, by Theorem 2 we obtain

$$
\begin{aligned}
\widehat{C_{X}}(u)= & \lambda \psi(u, 0) \frac{\mathrm{i}}{u} \sum_{j=1}^{n}\left(\hat{F}\left(u g\left(t_{j}-\right)\right)-\hat{F}\left(u g\left(t_{j}+\right)\right)\right) \\
& +\lambda \psi(u, 0) \frac{1}{\mathrm{i} u} \sum_{\left\{t_{j}: \Delta g\left(t_{j}\right)>0\right\}}\left(\hat{F}\left(u g\left(t_{j}+\right)\right)-\hat{F}\left(u g\left(t_{j}-\right)\right)\right) \\
& +\frac{1}{\mathrm{i} u} \sum_{\left\{t_{j}: \Delta g\left(t_{j}\right)<0\right\}}\left(\hat{F}\left(u g\left(t_{j}-\right)\right)-\hat{F}\left(u g\left(t_{j}+\right)\right)\right) \\
= & 2 \lambda \psi(u, 0) \frac{1}{\mathrm{i} u} \sum_{\left\{t_{j}: \Delta g\left(t_{j}\right)>0\right\}}\left(\hat{F}\left(u g\left(t_{j}+\right)\right)-\hat{F}\left(u g\left(t_{j}-\right)\right)\right) .
\end{aligned}
$$

This completes the proof of (9). Equation (10) follows from Remark 2, completing the proof of the proposition.

A particular case of Proposition 3 is obtained by making the additional assumption that the function $g$ is positive and that it has only one positive jump at $t_{1}=0$ from the value $g(0-)=0$ to the value $g(0+)>0$. Equation (9) then simply becomes

$$
\widehat{C_{X}}(u)=2 \lambda \psi(u, 0) \frac{1}{\mathrm{i} u}(\hat{F}(u g(0+))-1) .
$$

This framework corresponds to the case studied by Hsing [11], who considered upcrossings that are defined in the following way: the point $t$ is an upcrossing of the level $\alpha$ by the process $X$ if it is a point of discontinuity of $X$ and if $X(t-) \leq \alpha$ and $X(t+)>\alpha$. Then, Hsing proved that the expected number of such points in $(0,1)$, denoted by $U_{X}^{(J)}(\alpha)$, is given by

$$
U_{X}^{(J)}(\alpha)=\lambda \mathrm{P}[\alpha-\beta g(0+)<X(0) \leq \alpha],
$$

where $\beta$ and $X(0)$ are taken to be independent. Note that the left strict inequality comes from the way Hsing defined upcrossings of the level $\alpha$. Now, let us consider the usual definition of upcrossings of the level $\alpha$ (see [7, p. 192]): the point $t$ is an upcrossing of the level $\alpha$ by the process $X$ if there exists $\varepsilon>0$ such that $X(s) \leq \alpha$ in $(t-\varepsilon, t)$ and $X(s) \geq \alpha$ in $(t, t+\varepsilon)$; let us denote by $U_{X}(\alpha)$ their expected number in $(0,1)$. In a similar way we can define downcrossings of the level $\alpha$. Let us remark that, since $X$ is piecewise nonincreasing, if moreover $X$ is a.s. not identically equal to $\alpha$ in any interval of $(0,1)$ (this is satisfied, for instance, when $\mathrm{P}[X(0)=\alpha]=0)$, then the crossings of the level $\alpha$ are either upcrossings as defined by Hsing or downcrossings of the level $\alpha$. Moreover, by stationarity of the process $X$, the expected number of downcrossings of the level $\alpha$ is equal to $U_{X}(\alpha)$ and the result of Hsing yields

$$
C_{X}(\alpha)=2 U_{X}(\alpha)=2 \lambda \mathrm{P}[\alpha-\beta g(0+)<X(0) \leq \alpha] .
$$


The result of Hsing given in (12) is stronger than the similar formula (10) because his formula is valid for all levels $\alpha$ and moreover he needed weaker assumptions on the regularity of $g$. On the other hand, his proof strongly relied on the facts that $g$ has only one jump and that $g$ is identically 0 before that jump, and, thus, it cannot be generalized to other kernel functions $g$.

Finally, let us end this section by mentioning that we have studied here the case of a piecewise nonincreasing kernel function $g$, but that, of course, similar results hold for a piecewise nondecreasing kernel.

\section{High intensity and Gaussian limit}

We assume here that the assumptions of Proposition 2 hold. It is then well known (see [10] and [15] for instance) that, as the intensity $\lambda$ of the Poisson point process goes to infinity, the normalized process $Z_{\lambda}$ defined by

$$
t \mapsto Z_{\lambda}(t)=\frac{X_{\lambda}(t)-\mathrm{E}\left(X_{\lambda}(t)\right)}{\sqrt{\lambda}},
$$

where $X_{\lambda}$ denotes a shot noise process (as defined by (1)) with intensity $\lambda$ for the homogeneous Poisson point process, converges to a centered Gaussian process with covariance $R(t)=$ $\mathrm{E}\left(\beta^{2}\right) \int_{\mathbb{R}} g(s) g(s-t) \mathrm{d} s$.

Now, how does the number of level crossings of $Z_{\lambda}$ behave as $\lambda$ goes to $+\infty$ ? To answer this, we first determine the asymptotic expansion of the Fourier transform of $C_{Z_{\lambda}}$ as $\lambda \rightarrow+\infty$.

For $u \in \mathbb{R}$, we have

$$
\begin{aligned}
\widehat{C_{Z_{\lambda}}}(u)= & \frac{1}{\sqrt{\lambda}} \widehat{C_{X_{\lambda}}}\left(\frac{u}{\sqrt{\lambda}}\right) \mathrm{e}^{-\mathrm{i} u \mathrm{E}\left(X_{\lambda}(t)\right) / \sqrt{\lambda}} \\
= & \frac{1}{\sqrt{\lambda}} \mathrm{E}\left(\mathrm{e}^{\mathrm{i} u\left(X_{\lambda}(0)-\mathrm{E}\left(X_{\lambda}(0)\right)\right) / \sqrt{\lambda}}\left|X_{\lambda}^{\prime}(0)\right|\right) \\
& +\frac{\lambda}{\mathrm{i} u} \mathrm{E}\left(\mathrm{e}^{\mathrm{i} u\left(X_{\lambda}(0)-\mathrm{E}\left(X_{\lambda}(0)\right)\right) / \sqrt{\lambda}}\right) \sum_{j=1}^{n}\left(\mathrm{E}\left(\mathrm{e}^{\mathrm{i} u(\beta g)^{*}\left(t_{j}\right) / \sqrt{\lambda}}\right)-\mathrm{E}\left(\mathrm{e}^{\mathrm{i} u(\beta g)_{*}\left(t_{j}\right) / \sqrt{\lambda}}\right)\right) .
\end{aligned}
$$

From Section 4 of [3] we know that the first term on the right-hand side admits a limit as $\lambda \rightarrow+\infty$, and, more precisely, as $\lambda \rightarrow+\infty$,

$$
\frac{1}{\sqrt{\lambda}} \mathrm{E}\left(\mathrm{e}^{\mathrm{i} u\left(X_{\lambda}(0)-\mathrm{E}\left(X_{\lambda}(0)\right)\right) / \sqrt{\lambda}}\left|X_{\lambda}^{\prime}(0)\right|\right)=\sqrt{\frac{2 m_{2}}{\pi}} \mathrm{e}^{-m_{0} u^{2} / 2}+o(1),
$$

where $m_{0}=\mathrm{E}\left(\beta^{2}\right) \int_{\mathbb{R}} g^{2}(s) \mathrm{d} s$ and $m_{2}=\mathrm{E}\left(\beta^{2}\right) \int_{\mathbb{R}} g^{\prime 2}(s) \mathrm{d} s$. The second term is the product of two terms and each admits an asymptotic expansion as $\lambda \rightarrow+\infty$. Indeed, assuming moreover that $g \in L^{3}(\mathbb{R})$ and $\mathrm{E}\left(|\beta|^{3}\right)<\infty$, we have

$$
\begin{aligned}
\mathrm{E}\left(\mathrm{e}^{\mathrm{i} u\left(X_{\lambda}(0)-\mathrm{E}\left(X_{\lambda}(0)\right)\right) / \sqrt{\lambda}}\right) & =\exp \left(\lambda \int_{\mathbb{R}}\left(\hat{F}\left(\frac{u}{\sqrt{\lambda}} g(s)\right)-1\right) \mathrm{d} s-\mathrm{i} u \sqrt{\lambda} \mathrm{E}(\beta) \int_{\mathbb{R}} g(s) \mathrm{d} s\right) \\
& =\exp \left(-\frac{m_{0} u^{2}}{2}-\frac{\mathrm{i} m_{3} u^{3}}{3 \sqrt{\lambda}}+o\left(\frac{1}{\sqrt{\lambda}}\right)\right) \\
& =\mathrm{e}^{-m_{0} u^{2} / 2}\left(1+\frac{2 \mathrm{i} u m_{3}}{3 m_{0} \sqrt{\lambda}}\right)+o\left(\frac{1}{\sqrt{\lambda}}\right),
\end{aligned}
$$


where $m_{3}=\mathrm{E}\left(\beta^{3}\right) \int_{\mathbb{R}} g^{3}(s) \mathrm{d} s$. For a given jump number $j, 1 \leq j \leq n$, we have

$$
\begin{aligned}
& \frac{\sqrt{\lambda}}{\mathrm{i} u}\left(\mathrm{E}\left(\mathrm{e}^{\mathrm{i} u(\beta g)^{*}\left(t_{j}\right) / \sqrt{\lambda}}\right)-\mathrm{E}\left(\mathrm{e}^{\mathrm{i} u(\beta g)_{*}\left(t_{j}\right) / \sqrt{\lambda}}\right)\right) \\
& \quad=\mathrm{E}(|\beta|)\left|\Delta g\left(t_{j}\right)\right|+\frac{\mathrm{i} u}{2 \sqrt{\lambda}} \mathrm{E}\left(\beta^{2}\right)\left|\Delta g^{2}\left(t_{j}\right)\right|+o\left(\frac{1}{\sqrt{\lambda}}\right) .
\end{aligned}
$$

Finally, we thus have

$$
\begin{aligned}
\widehat{C_{Z_{\lambda}}}(u)= & \sqrt{\lambda} \mathrm{e}^{-m_{0} u^{2} / 2} \mathrm{E}(|\beta|) \sum_{j=1}^{n}\left|\Delta g\left(t_{j}\right)\right| \\
& +\left(\sqrt{\frac{2 m_{2}}{\pi}}+\frac{2 \mathrm{i} u m_{3}}{3 m_{0}} \mathrm{E}(|\beta|) \sum_{j=1}^{n}\left|\Delta g\left(t_{j}\right)\right|+\frac{\mathrm{i} u}{2} \mathrm{E}\left(\beta^{2}\right) \sum_{j=1}^{n}\left|\Delta g^{2}\left(t_{j}\right)\right|\right) \mathrm{e}^{-m_{0} u^{2} / 2} \\
& +o(1) .
\end{aligned}
$$

Let us comment on this result. When there are no jumps, $\widehat{C_{Z_{\lambda}}}(u)$ converges, as $\lambda$ goes to $\infty$, to $\left(\sqrt{2 m_{2} / \pi}\right) \mathrm{e}^{-m_{0} u^{2} / 2}$. This implies that $C_{Z_{\lambda}}(\alpha)$ weakly converges to $\left(\sqrt{m_{2}} / \pi \sqrt{m_{0}}\right) \mathrm{e}^{-\alpha^{2} / 2 m_{0}}$, which is the usual Rice formula for the mean number of level crossings of Gaussian processes (see [7, p. 194] for instance). Now, when there are jumps, the behavior of $\widehat{C_{Z_{\lambda}}}(u)$ is different, since the main term in $\sqrt{\lambda}$ does not vanish anymore. More precisely, $\widehat{C_{Z_{\lambda}}}(u) / \sqrt{\lambda}$ goes to $\mathrm{e}^{-m_{0} u^{2} / 2} \mathrm{E}(|\beta|) \sum_{j=1}^{n}\left|\Delta g\left(t_{j}\right)\right|$, which implies that

$$
\frac{1}{\sqrt{\lambda}} C_{Z_{\lambda}}(\alpha) \rightarrow \frac{1}{\sqrt{2 \pi m_{0}}} \mathrm{e}^{-\alpha^{2} / 2 m_{0}} \mathrm{E}(|\beta|) \sum_{j=1}^{n}\left|\Delta g\left(t_{j}\right)\right| \quad \text { as } \lambda \rightarrow \infty
$$

in the sense of weak convergence.

Note also that taking $u=0$ in the asymptotic formula for $\widehat{C_{Z_{\lambda}}}(u)$ gives the asymptotic behavior of the total variation of $Z_{\lambda}$. Indeed, we then have

$$
\mathrm{E}\left(\mathrm{TV}\left(Z_{\lambda},(0,1)\right)\right)=\widehat{C_{Z_{\lambda}}}(0)=\sqrt{\lambda} \mathrm{E}(|\beta|) \sum_{j=1}^{n}\left|\Delta g\left(t_{j}\right)\right|+\sqrt{\frac{2 m_{2}}{\pi}}+o(1) .
$$

This kind of asymptotic behavior has also been studied by Galerne [9] in the framework of random fields of bounded variation.

\section{Some examples}

\subsection{Step functions}

We start this section with some examples of explicit computations in the case of step functions.

Example 1. Consider the simple example of a step function where the kernel $g$ is a rectangular function: $g(t)=\mathbf{1}_{[0, a]}(t)$ with $a>0$. Note that this is a very simple framework, which also fits in the results of Hsing [11]. In this particular case $X$ is piecewise constant a.s.

- If $\beta=1$ a.s., we will prove that

$$
C_{X}(\alpha)=\sum_{k=0}^{+\infty} 2 \lambda \mathrm{e}^{-\lambda a} \frac{(\lambda a)^{k}}{k !} \mathbf{1}_{(k, k+1)}(\alpha) \quad \text { for all } \alpha \in \mathbb{R} \backslash \mathbb{N} .
$$


Note that $X$ takes values in $\mathbb{N}$ a.s., so $C_{X}$ is constant on any interval $(k, k+1)$ with $k \in \mathbb{N}$. Moreover, $\psi(u, v)=\exp \left(\lambda a\left(\mathrm{e}^{\mathrm{i} u}-1\right)\right)$, which shows, on the one hand, that $X(0)$ follows a Poisson distribution with parameter $\lambda a$ so that $C_{X}(\alpha)=+\infty$ for all $\alpha \in \mathbb{N}$. On the other hand, by (11),

$$
\widehat{C_{X}}(u)=2 \lambda \exp \left(\lambda a\left(\mathrm{e}^{\mathrm{i} u}-1\right)\right) \frac{\mathrm{e}^{\mathrm{i} u}-1}{\mathrm{i} u} .
$$

We recognize here the product of two Fourier transforms, a Poisson distribution and the indicator function of $[0,1]$, from which we deduce (14).

- If the impulse $\beta$ follows an exponential distribution of parameter $\mu>0$ then $\hat{F}(u)=$ $\mu /(\mu-\mathrm{i} u)$ and a simple computation gives $\psi(u, v)=\exp (\lambda a \mathrm{i} u /(\mu-\mathrm{i} u))$. On the one hand, the law of $X(0)$ can be computed as

$$
P_{X(0)}(\mathrm{d} x)=\mathrm{e}^{-2 a \lambda} \delta_{0}(\mathrm{~d} x)+\sum_{k=1}^{+\infty} \mathrm{e}^{-a \lambda} \frac{(2 a \lambda)^{k}}{k !} f_{\mu, k}(x) \mathrm{d} x,
$$

where $f_{\mu, k}$ is the probability density of the gamma distribution of parameters $\mu$ and $k$, and $\delta_{0}$ is the Dirac measure at point 0 . Then, $\mathrm{P}[X(0)=0]>0$ so that $C_{X}(0)=+\infty$ and, for all $\alpha>0, \mathrm{P}[X(0)=\alpha]=0$ so that, according to (13), $C_{X}$ is continuous on $(0,+\infty)$. On the other hand,

$$
\widehat{C_{X}}(u)=2 \lambda \exp \left(\lambda a \frac{\mathrm{i} u}{\mu-\mathrm{i} u}\right) \frac{1}{\mu-\mathrm{i} u} .
$$

We recognize here the Fourier transform of a noncentral chi-square distribution, and, thus, for all $\alpha>0$,

$$
C_{X}(\alpha)=2 \lambda \mu \mathrm{e}^{-a \lambda-\mu \alpha} I_{0}(2 \sqrt{a \lambda \mu \alpha}),
$$

where $I_{0}$ is the modified Bessel function of the first kind of order 0 ; it is given by

$$
I_{0}(x)=\frac{1}{\pi} \int_{0}^{\pi} \mathrm{e}^{x \cos \theta} \mathrm{d} \theta=\sum_{m=0}^{+\infty} \frac{x^{2 m}}{4^{m}(m !)^{2}} .
$$

Example 2. Consider a 'double rectangular' function given by $g(t)=\mathbf{1}_{[-1,0)}(t)-\mathbf{1}_{[0,1)}(t)$. Note that this case does not fit into the framework of Hsing [11]. However, $X$ is still piecewise constant a.s.

- If $\beta=1$ a.s. then simple computations show that

$$
\widehat{C_{X}}(u)=4 \lambda \frac{\sin u}{u} \exp (2 \lambda(\cos u-1)) .
$$

The last term above is the characteristic function of the difference of two independent Poisson random variables of the same parameter $\lambda$ (it is also called a Skellam distribution). Thus, as previously, we obtain $C_{X}(\alpha)=+\infty$ for all $\alpha \in \mathbb{Z}$ and, for all $\alpha \in \mathbb{R} \backslash \mathbb{Z}$,

$$
C_{X}(\alpha)=\sum_{k=-\infty}^{+\infty} 4 \lambda p_{k} \mathbf{1}_{(k, k+1)}(\alpha)
$$


where, for all $k \in \mathbb{Z}$,

$$
p_{k}=\mathrm{e}^{-2 \lambda} \sum_{n=0}^{+\infty} \frac{\lambda^{k+2 n}}{n !(k+n) !} .
$$

- If $\beta$ follows an exponential distribution of parameter $\mu$, we can also explicitly compute

$$
\widehat{C_{X}}(u)=\frac{4 \lambda \mu}{\mu^{2}+u^{2}} \exp \left(-2 \lambda \frac{u^{2}}{\mu^{2}+u^{2}}\right) .
$$

\subsection{Exponential kernel}

In this subsection we consider an example that has been widely studied in the literature: the impulse $\beta$ follows an exponential distribution of parameter $\mu>0$, and the kernel function $g$ is given by $g(t)=\mathrm{e}^{-t} \mathbf{1}_{[0,+\infty)}(t)$.

A simple computation gives the joint characteristic function of $X(0)$ and $X^{\prime}(0)$ as

$$
\psi(u, v)=\frac{\mu^{\lambda}}{(\mu-\mathrm{i} u+\mathrm{i} v)^{\lambda}} \quad \text { for all } u, v \in \mathbb{R} .
$$

Then, on the one hand, $X(0)$ follows a gamma distribution so that $\mathrm{P}[X(0)=\alpha]=0$ for all $\alpha \in \mathbb{R}$, and $C_{X}$ is a continuous function on $\mathbb{R}$ according to (13). On the other hand, by (11) we obtain

$$
\widehat{C_{X}}(u)=\frac{2 \lambda \mu^{\lambda}}{(\mu-\mathrm{i} u)^{\lambda+1}} .
$$

We recognize here the Fourier transform of another gamma probability density. Thus,

$$
C_{X}(\alpha)=\frac{2 \lambda \mu^{\lambda} \alpha^{\lambda} \mathrm{e}^{-\mu \alpha}}{\Gamma(\lambda+1)} \mathbf{1}_{[0,+\infty)}(\alpha) \quad \text { for all } \alpha \in \mathbb{R} .
$$

In the case where $\lambda$ is an integer, an explicit formula for the mean number of level crossings $C_{X}(\alpha)$ was given by Orsingher and Battaglia [14] (but they used a completely different approach based on the property that in the particular case of an exponential kernel, the process is Markovian). Finally, let us also mention the work of Borovkov and Last [4], who gave a version of Rice's formula for the mean number of level crossings in the framework of piecewisedeterministic Markov processes with jumps.

\section{Acknowledgements}

This work was supported by the French National Research Agency, under grant ANR-09BLAN-0029-01. We would also like to thank the anonymous referee for valuable comments and suggestions.

\section{References}

[1] Azais, J.-M. And Wschebor, M. (2009). Level Sets and Extrema of Random Processes and Fields. John Wiley, Hoboken, NJ.

[2] Bar-David, I. AND Nemirovsky, A. (1972). Level crossings of nondifferentiable shot processes. IEEE Trans. Inf. Theory 18, 27-34.

[3] Biermé, H. And Desolneux, A. (2012). Crossings of smooth shot noise processes. To appear in Ann. Appl. Prob.

[4] Borovkov, K. And Last, G. (2008). On level crossings for a general class of piecewise-deterministic Markov processes. Adv. Appl. Prob. 40, 815-834. 
[5] Breton, J.-C. (2010). Regularity of the laws of shot noise series and of related processes. J. Theoret. Prob. 23, 21-38.

[6] Choski, R. And Fonseca, I. (1997). A change of variables formula for mappings in BV. Proc. Amer. Math. Soc. 125, 2065-2072.

[7] Cramér, H. and Leadbetter, M. R. (2004). Stationary and Related Stochastic Processes. Dover, Mineola, NY.

[8] Evans, L. C. AND GARIEPY, R. F. (1992). Measure Theory and Fine Properties of Functions. CRC Press, Boca Raton, FL.

[9] Galerne, B. (2010). Stochastic image models and texture synthesis. Doctoral Thesis, Ecole Normale Supérieure de Cachan.

[10] Heinrich, L. AND SChmidt, V. (1985). Normal convergence of multidimensional shot noise and rates of this convergence. Adv. Appl. Prob. 17, 709-730.

[11] Hsing, T. (1987). On the intensity of crossings by a shot noise process. Adv. Appl. Prob. 19, 743-745.

[12] Leadbetter, M. R. (1966). On crossings of levels and curves by a wide class of stochastic processes. Ann. Math. Statist. 37, 260-267.

[13] Milnor, J. W. (1965). Topology from the Differential Viewpoint. The University Press of Virginia, Charlottesville, VA.

[14] Orsingher, E. and Battaglia, F. (1982). Probability distributions and level crossings of shot noise models. Stochastics 8, 45-61.

[15] Papoulis, A. (1971). High density shot noise and Gaussianity. J. Appl. Prob. 8, 118-127.

[16] Rice, J. (1977). On generalized shot noise. Adv. Appl. Prob. 9, 553-565.

[17] RyCHLIK, I. (2000). On some reliability applications of Rice's formula for the intensity of level crossings. Extremes 3, 331-348. 